\title{
Efecto del tiempo de reposo en la cinética de deshidratación de los granos de maíz nixtamalizados
}

\author{
María T. Zagaceta ${ }^{1}$, Rosaura Palma², Karen A. Aguilar ${ }^{3}$ y José L. Fernández-Muñoz ${ }^{4}$ \\ (1) Instituto Politécnico Nacional, Escuela Superior de Ingeniería Mecánica y Eléctrica Unidad Azcapotzalco, Ciudad de \\ México, México. (correo-e: mzagaceta@ipn.mx) \\ (2) Instituto Politécnico Nacional, Escuela Superior de Cómputo, Ciudad de México, México. (correo-e: rpalma@ipn.mx) \\ (3) Instituto Politécnico Nacional, Centro de Investigación en Computación, México Ciudad de México, México. \\ (correo-e: karen_ali320@hotmail.com) \\ (4) Instituto Politécnico Nacional, Centro de Investigación en Ciencia Aplicada y Tecnología Avanzada Unidad Legaria, \\ Ciudad de México, México. (correo-e: jlfernandez@ipn.mx)
}

* Autor a quien debe ser dirigida la correspondencia.

Recibido Nov. 29, 2019; Aceptado Ene. 27, 2020; Versión final Mar. 10, 2020, Publicado Jun. 2020

\begin{abstract}
Resumen
El objetivo de este trabajo fue estudiar y modelar la cinética de deshidratación de los granos de maíz nixtamalizados con diferente tiempo de reposo (TR) en su agua de cocción-alcalina. Estos granos con TR pierden humedad $(\mathrm{PH})$ continuamente un diferencial "dM" por diferencial de tiempo "dt", ambos son proporcionales a la cantidad de humedad $\mathrm{M}(\mathrm{t})$ existente en el grano de maíz hidratado a un tiempo " $\mathrm{t}$ " más una constante" C" (determinada con la aplicación de las condiciones de frontera). "K" es la contante de proporcionalidad. La ecuación diferencial propuesta tiene estructura matemática: $\mathrm{dM} / \mathrm{dt}=-\mathrm{K}[\mathrm{M}(\mathrm{t})+\mathrm{C}]$. Que al resolver esta ecuación diferencial y sustituir las condiciones de frontera la solución fue: $M(t)=\left(M_{0}-M_{E}\right) e^{K t}$ $\mathrm{ME}_{\mathrm{E}}$. En conclusión, este estudio presenta una hipótesis matemática para está formula que han utilizado muchos autores en sus publicaciones sin tener una hipótesis matemática.
\end{abstract}

\section{Effect of steeping time on dehydration kinetics of nixtamalized corn kernels}

\begin{abstract}
The objective of this work was to study and model the dehydration kinetics of nixtamalized corn kernels with different steeping time (ST) in cooking-alkaline water. These corn kernels with ST lose moisture (LM) continuously by differential "dM" by time differential "dt." Both are proportional to the amount of moisture M (t) existing in hydrated corn kernels at time "t" plus a constant " $\mathrm{C}$ " (determined with the application of boundary conditions). " $\mathrm{K}$ " is the proportionality constant. The proposed differential equation has the mathematical structure: $\mathrm{dM} / \mathrm{dt}=-\mathrm{K}[\mathrm{M}(\mathrm{t})+\mathrm{C}]$. When solving this differential equation and replacing boundary conditions, the solution was: $M(t)=\left(M_{0}-M_{E}\right) e^{K t}-M_{E}$. In conclusion, this study presents a mathematical hypothesis for this formula that many authors have used in their publications without having a mathematical hypothesis.
\end{abstract}




\section{INTRODUCCIÓN}

El maíz (Zea mays L.) es el tercer cereal más importante para el consumo humano en el mundo después del trigo y el arroz; es, y seguirá siendo un alimento básico en muchas regiones de América, África, entre otras. Se estima que, en 2012, la producción mundial de maíz fue de alrededor de 900 millones de toneladas, siendo los Estados Unidos de América, China, Brasil y Argentina los principales países productores de maíz (FAO, 2013). La industria de la tortilla representa aproximadamente una quinta parte del mercado general de maíz de México, con una producción anual estimada en 11.5 millones de toneladas, produciendo un valor de ventas de 7 mil millones de dólares. Cada mexicano consume un promedio de 90 kilogramos de tortilla por año (SIAP, 2008). El proceso de nixtamalización tradicional originario de México es un proceso hidrotérmico alcalino que consiste en la cocción de granos de maíz en solución de acuosa con hidróxido de calcio, con el objetivo principal de degradar el pericarpio para hacer más rápida la hidratación del grano en su totalidad acrecentando la difusión de iones calcio e iniciar con la pregelatinizar los almidones del endospermo para facilitar la molienda de los granos de maíz nixtamalizados durante su conversión en masa fresca.

Se define nixtamalización igual a la cocción alcalina de hidróxido de calcio $(0.8$ a $2 \% \mathrm{~m} / \mathrm{m})$ más un TR en su misma agua de cocción (Amador et al. 2019; Rangel-Hernández et al., 2019; Ramírez-Araujo et al., 2019; Castro-Muñoz et al., 2019) a una temperatura (de $72^{\circ} \mathrm{C}$ hasta cercano al punto de ebullición) y se continua con el tiempo de reposo (TR) en su licor de cocción (o nejayote Fernández-Muñoz et al., 2002) es una etapa de tratamiento térmico durante un enfriamiento desde la temperatura de cocción hasta la temperatura ambiente de los granos de maíz cocidos se conoce como tiempo de reposo (TR). Se drena el licor de cocciónreposo a los granos de maíz y se continua con el lavado tres veces con agua destilada para quitar el exceso de hidróxido de calcio principalmente, y finalmente podemos molerlos en un molino de piedras para convertirlos en masa fresca, la cual se moldea en forma de discos y al aplicar temperatura de cocción, la transformándolo en tortillas. También la masa fresca puede ser primero se deshidrata y segundo molida en un molino de martillo para convertirla en harina de maíz instantánea con un tamaño de partícula óptimo (malla 30 U.S de acuerdo con Fernández-Muñoz et al., 2016).

Las cinéticas de hidratación de los granos de maíz nixtamalizados (con cocción alcalina y TR) es una información muy importante analizar, debido a reflejará de alguna manera el estado fisicoquímico de cómo se encuentran las moléculas de agua y la facilidad de extraerlas al aplicarle una temperatura menor al de las transiciones químicas de los almidones como es la gelatinización. Destacando al binomio de las fases de cocción y reposo es lo que se conoce como proceso de nixtamalización tradicional del maíz. En estas fases de tiempo de cocción y reposo alcalino, ocurre de manera simultánea los procesos de la hidratación y pregelatinización parcial de los almidones del grano de maíz los cuales permitirá que los granos de maíz convertirlo en masa fresca, de manera paralela también se tiene el proceso de difusión de los iones de calcio máximamente durante el TR que van a determinar las propiedades finales fisicoquímicas, mecánicas y sensoriales del producto final.

El proceso de hidratación de los granos de maíz ha sido investigado por varios autores, donde calcularon la hidratación del grano de maíz variedad toluqueño con un periodo de calentamiento de 13 minutos a $92{ }^{\circ} \mathrm{C}$ como función del tiempo (Fernández et al., 2007). Existen diversos estudios publicados sobre el proceso de deshidratación de la masa o maíz nixtamalizado referidos en este trabajo de investigación. Se han estudiado el efecto del TR de 0 a $24 \mathrm{~h}$ sobre diferentes propiedades físicas-químicas de la harina de maíz preparada por el proceso de nixtamalización tradicional, así como en condiciones de sobresaturación de iones de calcio (Fernández-Muñoz, et al., 2002). Más recientemente, se evaluaron las propiedades reológicas de trece muestras de harina de maíz colectadas de varios productores comerciales de México (Núñez-Santiago, et al., 2003). Estas muestras se deshidrataron en un horno de convección a $45^{\circ} \mathrm{C}$. También se reporta el proceso de difusión de iones de calcio a través de las diferentes partes de granos de maíz (el pericarpio, el endospermo y el germen) durante el proceso de nixtamalización tradicional en función del TR de 0 a 24 h (FernándezMuñoz, et al., 2004). En este experimento el pericarpio, el endospermo y el germen de cada grano reposado fueron separados manualmente y se deshidrataron en un horno con circulación de aire forzado a $40^{\circ} \mathrm{C}$ durante 6 h (cada pieza alcanzó un contenido de humedad relativa del 10\%). Para hacer el estudio de la difusión de iones de calcio a través de las diferentes zonas del grano de maíz durante la nixtamalización con microondas en función del TR ( 0 a 24 h), esto se llevó a cabo en dos procesos diferentes de cocimiento: el primero por 100 min a $72 \stackrel{\circ}{\circ}$ y el segundo por 45 min a $92{ }^{\circ} \mathrm{C}$ (Fernández-Muñoz, et al., 2006). Donde la cuantificación del porcentaje de calcio se hizo en: el pericarpio, el endospermo y el germen se hicieron en muestra de 200 g de maíz que fueron separados manualmente y se secaron en un horno de circulación de aire forzado a 40 ${ }^{\circ} \mathrm{C}$ durante $8 \mathrm{~h}$ (cada componente alcanza un contenido de humedad relativa del $12 \%$ ).

Se analizaron los cambios microestructurales en el pericarpio del maíz durante la cocción y TR alcalinahidrotermal en el proceso de nixtamalización tradicional (Rojas-Molina, et al., 2009). A las muestras de maíz le fueron separado el pericarpio y se deshidrató en una estufa de vacío a $40^{\circ} \mathrm{C}$ durante $6 \mathrm{~h}$, hasta alcanzar un contenido de humedad del $10 \%$. Se reporta el mecanismo de la absorción de calcio en el grano de maíz 
durante el proceso de nixtamalización (Valderrama-Bravo, et al., 2010). En este proceso la absorción de calcio se ha explicado también en términos del proceso de difusión de iones de calcio, aunque se han reportado que otros mecanismos como la acumulación de calcio en las capas externas del pericarpio, y los cambios físicos de la estructura del pericarpio se encuentran íntimamente relacionados con el fenómeno de la percolación contribuyendo a la absorción de calcio.

En otra investigación, la masa fresca de maíz nixtamalizado fue deshidratada en un secador flash y pulverizado utilizando un molino de martillos equipado con una malla de $0.8 \mathrm{~mm}$ o malla 30 U.S. (FernándezMuñoz et al., 2016). Las muestras fueron envasadas al vacío a $4{ }^{\circ} \mathrm{C}$ hasta el momento del análisis de espectrometría de absorción atómica. Cada muestra se prepara por separado de para diferentes tamaños de partícula como un evento independiente. A pesar de los diversos estudios realizados, no existen evidencias en los resultados que expliquen adecuadamente con modelos matemáticos el fenómeno de cinética de deshidratación en función del TR de los granos de maíz durante el tratamiento hidrotermal alcalino. Un hecho importante de enfatizar durante la elaboración de una la harina de maíz nixtamalizado es el porcentaje de humedad debe estar entre 10 a $15 \%$ para reducir el crecimiento de salmonela durante su almacenamiento (Van Cauwenberge, et al., 2003). Por esta razón tecnológica de conservación de alimentos es de vital importante estudiar la cinética de deshidratación de los granos de maíz nixtamalizados con TR y con conocer el efecto del TR durante el todo proceso de nixtamalización tradicional, con el estudio y análisis con un modelo matemático para su mayor juicio científico y aplicación tecnológica. El objetivo fue deducir matemáticamente la ecuación diferencial de primer orden que describa las cinéticas de deshidratación, establecer las condiciones de frontera que debe de cumplir de acuerdo a las observaciones experimentales, resolver la ecuación diferencial con el método de separación de variables e integración, y finalmente ajustar el modelo matemático a los datos experimentales para su validación durante el proceso de deshidratación de los granos de maíz con diferentes TR.

\section{METODOLOGÍA}

El proceso de nixtamalización tradicional se realiza con una mezcla de agua y granos de maíz $3: 1$ (volumen/peso) con una concentración de $\mathrm{Ca}(\mathrm{OH})_{2} \quad 0.8 \%$ en relación a $45 \mathrm{~g}$ de granos de maíz blanco ( $14.54 \%$ de humedad endógena) cada experimento se realiza por quintuplicado. Las muestras de maíz son cocidas en agua-maíz-Ca(OH) 2 a una temperatura de $80^{\circ} \mathrm{C}$ por 90 minutos y finalmente aplicando diferentes $\mathrm{TR}=0,2,4,6$, y $8 \mathrm{~h}$ en su licor de cocción (o nejayote). Posteriormente, se deshidratan a $50^{\circ} \mathrm{C}$ desde 0 a 120 minutos y 2600 minutos (para obtener el parámetro Me) en una balanza con lámpara de halógeno modelo HR83 Mettler-Toledo.

\section{Implementación de un Modelo Matemático}

En el proceso de deshidratación de los granos de maíz nixtamalizados pierde humedad continuamente hasta un porcentaje límite mejor conocida como la humedad de equilibrio $\mathrm{M}_{\mathrm{E}}$, por lo que la cantidad humedad perdida es un diferencial "dM" en un diferencial de tiempo "dt" ambos son proporcional al porcentaje de humidad $\mathrm{M}(\mathrm{t})$ existente en el grano de maíz hidratado a un tiempo " $\mathrm{t}$ " más una constante desconocida" $\mathrm{C}$ ", $\mathrm{dM}$ $\alpha-[M(t)+C] d t$, donde " $K$ " es la contante de proporcionalidad. El valor que tome la constante " $C$ " ayudará a que el modelo matemático converja y así describa la cinética de deshidratación para todo tiempo de reposo de deshidratación. Se propone el modelo en forma de teorema.

Teorema 1. La deshidratación $\mathrm{M}(\mathrm{t})$ de los granos de maíz nixtamalizados puede ser expresada como una ecuación (1) diferencial de primer orden del tipo Ley de enfriamiento de Newton, se tiene:

$$
\frac{d M(t)}{d t}=-K[M(t)+C]
$$

Considerando los límites o condiciones de frontera dados por la ecuación (2) con base a lo observado experimentalmente se deben cumplir absolutamente son:

$$
M(t)=\left\{\begin{array}{l}
M_{0} \Leftrightarrow t \rightarrow 0 \\
M_{e} \Leftrightarrow t \rightarrow \infty
\end{array}\right.
$$

Se tiene la ecuación del modelo empírico de Page (3), con $n=1$ al considerar $M_{e}=M_{\infty}$ y la razón de humedad se expresa con la ecuación (3), la ecuación (3a) es la ecuación de Page de acuerdo con Giraldo-Zuniga et al,. (2010), al comparar las ecuaciones (3) y (3a) son iguales si y solo si cuando $n=1$ y K=k. 


$$
\begin{aligned}
& M R=\frac{M(t)-M_{e}}{M_{0}-M_{e}}=e^{-K t} \\
& M R=\frac{M(t)-M_{e}}{M_{0}-M_{e}}=e^{-\mathrm{k} t^{n}}
\end{aligned}
$$

La prueba del teorema: Integrar la ecuación (1) con el método de separación de variables y los límites de integración de $M_{0}$ (porcentaje de humedad inicial cuando el tiempo a cero minutos) hasta la $M(t)$ (porcentaje de humedad a un tiempo t).

$$
\int_{M_{0}}^{M(t)} \frac{d t}{M(t)+C}=-K \int_{0}^{t} d t
$$

Progreso de la integral (4) se obtiene (5):

$$
\ln \left(\frac{M(t)+C}{M_{0}+C}\right)=-K t
$$

Al despejar $\mathrm{M}(\mathrm{t})$ de (5) resulta (6) cuyo modelo es de la forma de la ecuación con una estructura matemática de enfriamiento de Newton:

$$
M(t)=\left(M_{0}+C\right) e^{-K t}-C
$$

Validando matemáticamente el cumplimiento de las condiciones iniciales (2) en la ecuación (6) se logra obtener las ecuaciones (7) y (8):

$$
\begin{aligned}
& \operatorname{Lim}_{t \rightarrow 0}\lfloor M(t)\rfloor=\operatorname{Lim}_{t \rightarrow 0}\left[\left(M_{0}+C\right) e^{-K t}-C\right]=M_{0} \\
& \operatorname{Lim}_{t \rightarrow \infty}\lfloor M(t)]=\operatorname{Lim}_{t \rightarrow \infty}\left[\left(M_{0}+C\right) e^{-K t}-C\right]=-C=M_{e}
\end{aligned}
$$

La ecuación (6) se transforma al sustituir la constante $C=-M_{e}$ en la ecuación (9) que describe el proceso de deshidratación que nos ocupa en este trabajo de investigación cuando sustituimos las ecuaciones (8) en (6):

$$
M(t)=\left(M_{0}-M_{e}\right) e^{-K t}+M_{e}
$$

La ecuación (9) fue propuesta por varios autores sin hacer su deducción matemática total y conocer su estructuración matemática experimental (Abu-McKenna, 1997; Haladjian et al., 2003), por lo que la ecuación (9) se hace un desarrollo matemático que respalda fuertemente el hacer este artículo de investigación con aportaciones reales al universo de los textos en la aplicación de las ecuaciones diferenciales; y con un procedimiento de cumplimiento de las condiciones iniciales al hacer un desarrollo lógico matemático experimental. Desde ahora tenemos una ecuación diferencial (9a) de primer orden que describe la cinética de deshidratación de los alimentos para que sea incorporada a los libros de texto de ecuaciones diferenciales en su sección de aplicaciones cuya solución es la ecuación (9).

$$
\frac{d M(t)}{d t}=-K\left[M(t)-M_{e}\right]
$$

Al despejarla el "t" de la ecuación (9) se obtiene la ecuación (10), para calcular el tiempo requerido para deshidratar las muestras a una humedad determinada $M(t)$ que se elija a una temperatura constante sin reacciones químicas.

$$
t=\frac{1}{K} \operatorname{Ln}\left[\frac{M_{0}-M_{e}}{M(t)-M_{e}}\right]=\frac{1}{K} \operatorname{Ln}\left[\frac{1}{M R}\right]
$$

La hidratación $\mathrm{M}(\mathrm{t})$ de los granos de maíz está representada por una ecuación de una variable (t) y tres los parámetros constantes $\left(\mathrm{M}_{0}, \mathrm{M}_{\mathrm{e}}, \mathrm{K}\right)$, las dos primeras constantes son obtenidas de las mediciones experimentales y la tercera del ajuste con la ecuación (9) con los datos experimentales. También podemos escribir así la razón de deshidratación en función del tiempo como la ecuación (11) y (12);

$$
M R=\frac{M(t)-M_{e}}{M_{0}-M_{e}}=e^{-K t}=\sum_{n=0}^{\infty} \frac{(-K t)^{n}}{n !}
$$




$$
1-M R=1-\frac{M(t)-M_{e}}{M_{0}-M_{e}}=\frac{M_{e}-M(t)}{M_{0}-M_{e}}=1-e^{-K t}=\sum_{n=1}^{\infty} \frac{(-K t)^{n}}{n !}
$$

La ecuación (11) se conoce por varios autores como la ecuación del modelo empírico de Page con $\mathrm{n}=1$ y Mo = Me (Miano, et al., 2017; Gocen, et al., 2007). Al Calcular la primera derivada de la ecuación (9) se obtiene la velocidad de deshidratación representado por la ecuación (13), donde el signo negativo tiene una representación física muy importante personificando la pérdida de humedad de las muestras para todo tiempo de deshidratación:

$$
\frac{d M(t)}{d t}=-K\left(M_{0}-M_{e}\right) e^{-K t}
$$

Las condiciones iniciales que debe de cumplir la ecuación (13) son propuestas con base a las observaciones experimentales representadas por la ecuación (14). Donde $V_{0}$ es la velocidad cuando el tiempo tiende a cero y debe de ser máxima por la alta disponibilidad de moléculas de agua que hidrataron el grano de maíz en sus etapas de cocción y TR:

$$
\frac{d M(t)}{d t}=\left\{\begin{array}{c}
V_{0} \Leftrightarrow t \rightarrow 0 \\
0 \Leftrightarrow t \rightarrow \infty
\end{array}\right.
$$

Por lo tanto, el cálculo matemático de la velocidad inicial es la ecuación (15) y depende de tres parámetros K que se calcula del ajuste matemático con los datos experimentales, $M_{0}$ y $M_{E}$ se obtuvo de los datos experimentales. Por último, la ecuación (16) información que arroja fue la velocidad tiende a cero cuando el tiempo tiende a infinito y es lo que se observa cuando deshidratamos un alimento.

$$
\begin{aligned}
& \operatorname{Lim}_{t \rightarrow 0}\left[\frac{d M(t)}{d t}\right]=\operatorname{Lim}_{t \rightarrow 0}\left[-K\left(M_{0}-M_{e}\right) e^{-K t}\right]=-K\left(M_{0}-M_{e}\right)=V_{0} \\
& \operatorname{Lim}_{t \rightarrow \infty}\left[\frac{d M(t)}{d t}\right]=\operatorname{Lim}_{t \rightarrow \infty}\left[-K\left(M_{0}-M_{e}\right) e^{-K t}\right]=0
\end{aligned}
$$

Adicionalmente, la ecuación (15) fue el cálculo de la velocidad inicial del proceso de deshidratación con un valor máximo debido al alto porcentaje de humedad y en cambio la ecuación (16) nos muestra que a medida que se incrementa el tiempo de deshidratación la velocidad disminuye cero al decaer el número de moléculas libres en la estructura del grano de maíz. Al demostrar que la ecuación (9) si cumple con las condiciones iniciales (14), por lo tanto, es un modelo propicio para describir la cinética de deshidratado y de igual manera poder calcular la velocidad cuando $t \rightarrow 0$ al aplicar el límite en la ecuación (13). Se está utilizando la fortaleza de los modelos matemáticos y así poder explicar ampliamente todo el proceso de deshidratación, como su velocidad inicial, fundamentado con las condiciones iniciales que se deben de cumplir. Los datos experimentales se analizaron estadísticamente con el Excel con el cálculo de la media con la ecuación (17) y la desviación típica muestra o valor de incertidumbre ecuación (18), donde n es el número de mediciones.

$$
\begin{aligned}
& \operatorname{Media}(M(t))=\frac{\sum_{i=1}^{N} M_{i}(t)}{n} \\
& S_{x}=\sqrt{\frac{\sum_{i=1}^{N}\left[M_{i}(t)-\operatorname{Media}\left(M_{i}(t)\right]^{2}\right.}{n-1}}
\end{aligned}
$$

\section{RESULTADOS Y DISCUSIÓN}

Observando los datos experimentales en la tabla 1 son la media (véase ecuación 17) de cinco mediciones del $\mathrm{PH}$ como una función del TD, con sus valores incertidumbre $\left( \pm \mathrm{S}_{\mathrm{x}}\right)$ en las mediciones para cada uno de los TR $(0,2,4,6$, y $8 \mathrm{~h})$. EI TD fue de 0 a $120 \mathrm{~min}$ con intervalos temporales de $15 \mathrm{~min}$ a $50^{\circ} \mathrm{C}$ permanente. Analizando los valores de incertidumbre (véase ecuación 18) para cada uno de los $\mathrm{PH}$ como una función del TD en los diferentes TR son: $0.352 \leq \pm S_{x} \leq 0.886$ para $0 h$ con máximo en 0 min y mínimo en 90 min; $0.373 \leq \pm S_{x} \leq 0.670$ para $2 \mathrm{~h}$ con máximo en 0 min y mínimo en $60 \mathrm{~min} ; 0.181 \leq \pm S_{x} \leq 0.667$ para $4 \mathrm{~h}$ con máximo en 15 min y mínimo en $120 \mathrm{~min} ; 0.501 \leq \pm S_{x} \leq 0.993$ para $6 \mathrm{~h}$ con máximo en 0 min y mínimo en $105 \mathrm{~min} ; 0.228 \leq \pm S_{x} \leq 0.697$ para 8h con máximo en 0 min y mínimo en 120 min 
Los valores máximos en la incertidumbre para $0,2,6$ y $8 \mathrm{~h}$ de TR se mantiene constante excepto para $8 \mathrm{~h}$ se recorre a $15 \mathrm{~min}$ de TD. Y valores mínimos en la incertidumbre fluctúa de 90 (0h TR) a 60(2h TR) a 120(4h TR) a 105(6h TR) y regresa nuevamente a 120(8h TR) min de TD. Lo más transcendental de estos datos estadísticos es que sus valores en la incertidumbre son pequeños, entonces cada punto experimental está bien representado matemáticamente vs experimentalmente. En la figura 1 se muestran los puntos de deshidratación de los granos de maíz cocidos en solución acuosa de hidróxido de calcio sin y con TR en su agua de cocción a 0, 2, 4, 6 y $8 \mathrm{~h}$, cada una de las muestras de maíz se deshidrata por quintuplicado a $50^{\circ} \mathrm{C}$, representando cada punto un promedio de cinco mediciones. Se hizo un análisis de los puntos de deshidratación, encontrando una variación en la pérdida del porcentaje humedad de manera significativa que lo avala la barra de incertidumbre al no tocarse una con otra, y subsiguientemente fue reduciendo con la forma de los perfiles de deshidratación se incrementó el TD aproximándose asintóticamente a la humedad de equilibrio $M_{E}$ como se definió en la referencia de Peleg, 1988. No existe diferencia significativa de los $M_{E}$ debido a que tiende a un valor asintótico único, otro punto experimental que avala el modelo matemático representado por las ecuaciones (9) y (9a). Se observa un efecto significativo en $M_{0}$ cuando se incrementa el TR y es lo que influye en el rendimiento de la masa después de molerlos los granos de maíz nixtamalizados en un molino de piedras y por lo tanto se reflejara en el rendimiento de las tortillas como producto final. Como se muestra en la Tabla 2 los parámetros obtenidos mediante el ajuste de la ecuación (9) con los datos experimentales del proceso de deshidratación de los granos de maíz nixtamalizados. Las formas de los puntos de deshidratación siguelan un perfil similar para todas las muestras con TR de 2, 4, 6, y 8 horas, incrementándose con el TR, exceptuando la muestra con solo cocción alcalñina; estos resultados de deshidratación tienen la misma forma similar que los ya reportados por Arámbula, et al., 1999. De igual manera observamos que durante la fase de TR se incrementó la hidratación de los granos de maíz de manera significativa, y está de acuerdo con lo ya publicado por varios autores (Fernández-Muñoz, et al., 2007; Laria, et al., 2005; Contreras-Jiménez, et al., 2014).

Tabla 1: La media del PH como una función del TD (a $\left.50^{\circ} \mathrm{C}\right)$ de los granos de maíz cocidos y con $0,2,4,6$, y 8 h de TR con su respectivo $S_{x} \pm$

\begin{tabular}{|l|l|l|l|l|l|}
\hline $\mathrm{T}=50^{\circ} \mathrm{C}$ & & $\begin{array}{l}\text { La media de M(t) } \\
(\%) \mathrm{m} / \mathrm{m}\end{array}$ & & \\
\hline $\mathrm{TD}(\mathrm{min})$ & $\mathrm{TR}=0 \mathrm{~h}\left( \pm \mathrm{S}_{\mathrm{x}}\right)$ & $\mathrm{TR}=2 \mathrm{~h}\left( \pm \mathrm{S}_{\mathrm{x}}\right)$ & $\mathrm{TR}=4 \mathrm{~h}\left( \pm \mathrm{S}_{\mathrm{x}}\right)$ & $\mathrm{TR}=6 \mathrm{~h}\left( \pm \mathrm{S}_{\mathrm{x}}\right)$ & $\mathrm{TR}=8 \mathrm{~h}\left( \pm \mathrm{S}_{\mathrm{x}}\right)$ \\
\hline 0 & $37.360 \pm 0.886$ & $46.394 \pm 0.376$ & $47.679 \pm 0.571$ & $49.098 \pm 0.993$ & $50.942 \pm 0.697$ \\
\hline 15 & $29.511 \pm 0.819$ & $39.020 \pm 0.490$ & $40.227 \pm 0.667$ & $41.934 \pm 0.728$ & $43.589 \pm 0.596$ \\
\hline 30 & $23.403 \pm 0.679$ & $31.887 \pm 0.633$ & $33.095 \pm 0.638$ & $34.899 \pm 0.646$ & $36.435 \pm 0.589$ \\
\hline 45 & $18.774 \pm 0.483$ & $25.714 \pm 0.630$ & $26.890 \pm 0.517$ & $28.794 \pm 0.566$ & $29.926 \pm 0.582$ \\
\hline 60 & $15.458 \pm 0.414$ & $20.732 \pm 0.670$ & $21.790 \pm 0.425$ & $23.659 \pm 0.615$ & $24.911 \pm 0.561$ \\
\hline 75 & $13.164 \pm 0.370$ & $16.992 \pm 0.600$ & $17.967 \pm 0.317$ & $19.585 \pm 0.597$ & $20.687 \pm 0.453$ \\
\hline 90 & $11.515 \pm 0.352$ & $14.409 \pm 0.430$ & $15.256 \pm 0.237$ & $16.627 \pm 0.539$ & $17.493 \pm 0.302$ \\
\hline 105 & $10.192 \pm 0.372$ & $12.383 \pm 0.445$ & $13.296 \pm 0.256$ & $14.365 \pm 0.501$ & $15.061 \pm 0.256$ \\
\hline 120 & $8.919 \pm 0.449$ & $11.063 \pm 0.471$ & $11.415 \pm 0.181$ & $12.392 \pm 0.541$ & $13.139 \pm 0.228$ \\
\hline 2600 & $5.088 \pm 0.705$ & $5.399 \pm 0.383$ & $5.792 \pm 0.456$ & $5.646 \pm 0.524$ & $5.836 \pm 0.494$ \\
\hline
\end{tabular}

Los datos experimentales están representados en la figura 1 con puntos geométricos de colores y el modelo matemático son las líneas continuas de color que pasa sobre los puntos experimentales del mismo color. Este modelo matemático transcendental es la ecuación (9) que fue ajustada a los datos experimentales, con lo cual se obtiene un valor de determinación entre $0.994 \leq R^{2} \leq 0.997$ con $P \leq 0.05$; con base en los valores de la determinación podemos validar matemáticamente que la ecuación (9) es adecuada para predecir las cinéticas de deshidratación de los granos de maíz cocido en solución acuosa de hidróxido de calcio sin y con TR en su agua de cocción, como lo podemos verificar como se muestran en la tabla 2. La hidratación de equilibrio se encuentra de $5.088 \leq M_{E} \leq 5.836$; la hidratación inicial se encuentra de $37.360 \leq M_{0} \leq 50.942$; constante cinética se encuentra de $54.080 \leq 1 / K \leq 69.321$; y $0.994 \leq R^{2} \leq 0.999$. Todos estos valores se incrementan en forma mayoritaria al aumentar el TR.

Tabla 2: Parámetros de la cinética de deshidratación del grano de maíz a $50^{\circ} \mathrm{C}$ de las ecuaciones (9) y (13)

\begin{tabular}{|l|l|l|l|l|}
\hline$T R$ & $M_{e} \% m / m$ & $M_{0} \% m / m$ & $1 / K$ & $R^{2}$ \\
\hline 0 & $5.088 \pm 0.705$ & $37.360 \pm 0.886$ & $53.14 \pm 3.17$ & $0.997 \pm 0.001$ \\
\hline 2 & $5.399 \pm 0.383$ & $46.394 \pm 0.376$ & $62.27 \pm 2.8$ & $0.995 \pm 0.001$ \\
\hline 4 & $5.792 \pm 0.456$ & $47.679 \pm 0.571$ & $63.55 \pm 1.7$ & $0.995 \pm 0.002$ \\
\hline 6 & $5.646 \pm 0.524$ & $49.098 \pm 0.993$ & $67.34 \pm 2.7$ & $0.996 \pm 0.001$ \\
\hline 8 & $5.836 \pm 0.494$ & $50.942 \pm 0.697$ & $69.34 \pm 2.26$ & $0.996 \pm 0.001$ \\
\hline
\end{tabular}


En la figura 2 se muestra los perfiles de la velocidad de deshidratación de granos de maíz cocidos y reposados en solución acuosa de hidróxido de calcio durante un periodo de 0 a $8 \mathrm{~h}$. La velocidad de deshidratación de los granos de maíz se incrementa con el acrecentamiento del TR al deshidratar a tiempos cortos, entonces la dependencia de la velocidad de deshidratación resulta inversamente proporcional al TR. Los parámetros que se exponen en la tabla 2 están de acuerdo con el modelo matemático ecuación (13). El signo menos representa físicamente la pérdida del $\mathrm{PH}$ en las muestras durante el proceso de deshidratación. Se puede apreciar que la velocidad es continua durante la deshidratación tiene una dependencia con el TR que se les aplica a las muestras de maíz incrementando su rendimiento en tortilla cuando pierden menos humedad durante el proceso de cocción de la misma, para completar el proceso de nixtamalización es cocción más reposo.

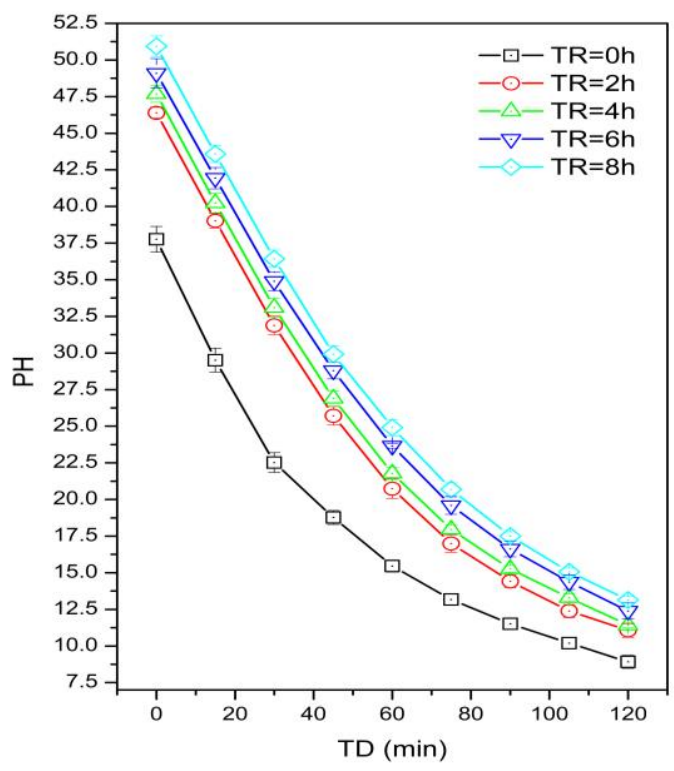

Fig. 1: Deshidratación de muestras en función del tiempo con y sin TR en su agua de cocción

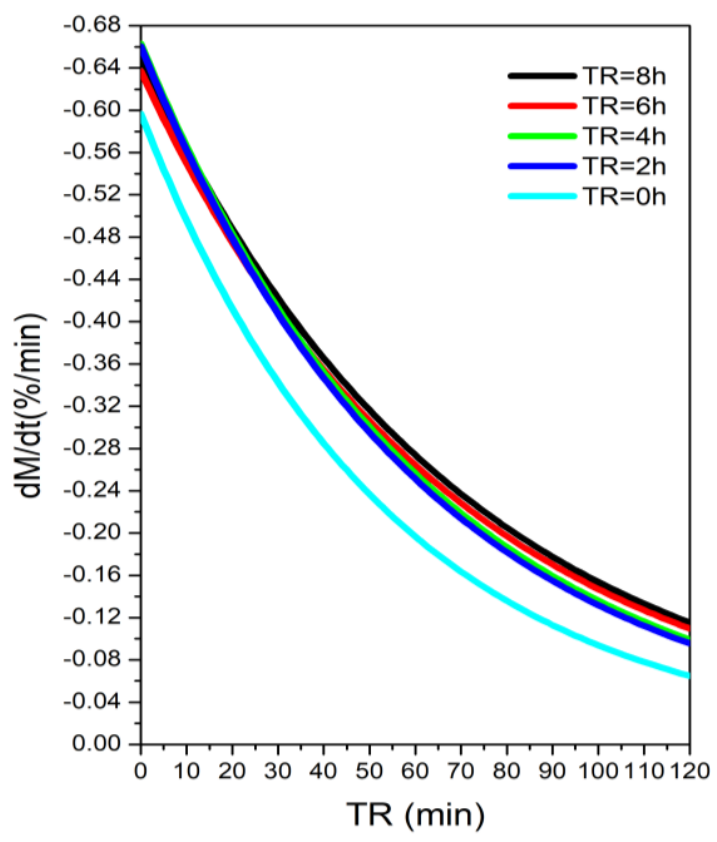

Fig. 2: Velocidad de deshidratación de granos de maíz con tratamiento hidrotérmico alcalinamente sin y con tiempo de reposo obtenidos con la ecuación (13) y los parámetros que se muestran en la tabla 2.

Una utilidad práctica tecnológica es el poder predecir el tiempo que necesitamos nixtamalizar (cocción más TR) los granos de maíz y deshidratar para que tenga la cantidad de humedad necesaria para elaborar el producto que deseamos. Es necesario conocer la dependencia entre el binomio TD vs TR para que tenga el $\mathrm{PH}$ deseado para elaborar un producto final, se despeja " $\mathrm{t}$ " de la ecuación (10) y se sustituye el valor de M(t) 
fijo, y así se calcula el tiempo a ocupar. Entonces para construir los datos de la fig. 3, se fijó $\mathrm{M}(\mathrm{t}) \operatorname{con}$ los 9 , $10,11,12$ y $13 \%$ en la ecuación (10) para cada uno de los TR se obtuvo un perfil no lineal en forma curva "S" que se desplaza en el eje de las ordenadas conforme disminuye el PH requerida como se muestran en la figura 3. Se requiere más TD cuando se incrementa el TR como lo podemos observar con los puntos de la derecha TD vs TR. Existe una dependencia no lineal entre la humedad fijada con el binomio TD vs. TR durante el tratamiento hidrotérmico alcalino aplicado a los granos de maíz durante el proceso de nixtamalización tradicional.

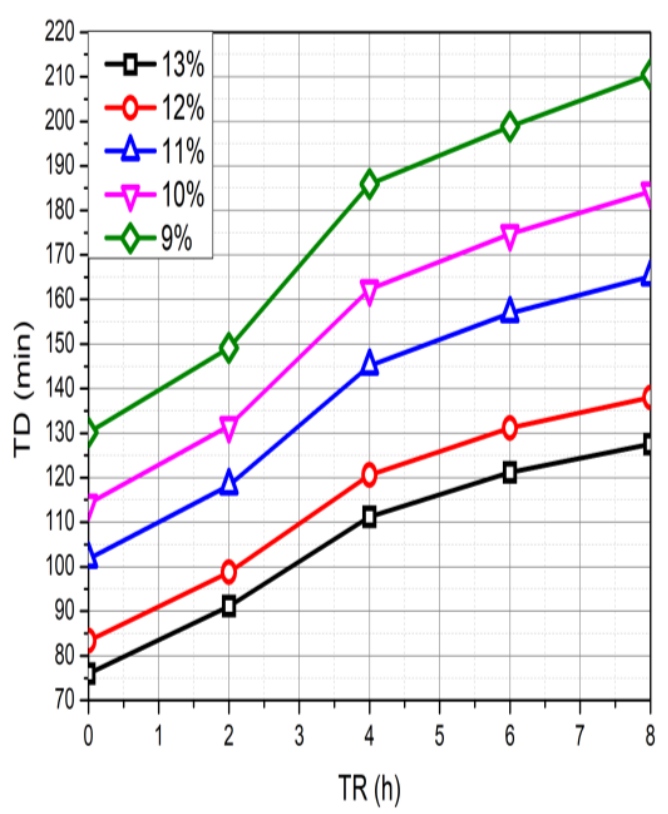

Fig. 3: tiempo de deshidratación de granos de maíz para obtener un producto final de $13,12,11,10$ y $9 \%$ de humedad para cada uno de los tiempos de reposo.

\section{CONCLUSIONES}

Con base en el estudio y análisis matemático realizado se pueden obtener las siguientes conclusiones esenciales: (i) Desde ahora tenemos la ecuación diferencial (9a) de primer orden que describe la cinética de deshidratación de los alimentos para que sea incorporada a los libros de texto de ecuaciones diferenciales en su sección de aplicaciones cuya solución es la ecuación (9) y las condiciones iniciales que cumple están representado por la ecuación (2), (ii) con la ecuación (10) podemos calcular el tiempo requerido para alcanzar el PH que fijamos, (iii) Con estos modelos matemáticos podemos predecir la humedad requerida para una posible molienda ya sea seca o húmeda según la aplicación tecnológica, (iv) La estructuración con fundamentos experimentales y matemáticos de las ecuaciones (9), (9a) y (2) es nuestro desarrollo más importante de este artículo, validándola fuertemente con una idea original de su planteamiento y demostración y (v) Una menor porcentaje deshidratación de los granos de maíz será reflejado en el rendimiento de las tortillas de maíz.

\section{AGRADECIMIENTOS}

Agradecemos a la Secretaría de Investigación y de Posgrado del Instituto Politécnico Nacional (SIP-IPN).

\section{REFERENCIAS}

Abu-Ghannam, N., y McKenna, B., Hydration kinetics of red kidney beans. https://doi.org/10.1111/j.13652621.1997.tb04420.x, J. Food Sci., 62(3), 520-523 (1997).

Amador-Rodríguez, K. W., Martínez-Bustos, F., y Silos-Espino, H., Effect of High-Energy Milling on Bioactive Compounds and Antioxidant Capacity in Nixtamalized Creole Corn Flours. https://doi.org/10.1007/s11130-019-00727-9, Plant Foods Human Nut, 74(2), 241-246 (2019).

Arambula, V.G., Mauricio, S.R.A., Figueroa, C.J.D., González-Hernández, J., y Ordorica. F.C.A., Corn Masa and Tortillas from Extruded Instant Corn Flour Containing Hydrocolloids and Lime. https://doi.org/10.1111/j.1365-2621.1999.tb09873.x, J. Food Sci. 64(1), 120-124 (1999).

Castro-Muñoz, R., Fíla V., y Durán-Páramo, E., A Review of the Primary By-product (Nejayote) of the Nixtamalization During Maize Processing: Potential Reuses. http://dx.doi.org/10.1007/s12649-017-0029-4, Waste Biomass Valor 10:1322, (2019) 
Contreras-Jiménez, B., Gaytán-Martínez, M., Figueroa-Cárdenas, J. D., Avalos-Zúñiga, R. A., y Morales-Sánchez, E., Effect of steeping time and calcium hydroxide concentration on the water absorption and pasting profile of corn grits. https://doi.org/10.1016/j.jfoodeng.2013.09.005, J. Food Eng. 122(1) 89, $72-77$ (2014).

FAO, Food and Agriculture Organization of the United Nations Statistics Division, http://faostat3.fao.org/browse/Q/QC/E. (2013).

Fernández-Muñoz, J. L., Rodríguez, M. E., Pless, R. C., Martínez-Flores, H. E., Leal, M., Martínez, J. L., y Baños, L., Changes in Nixtamalized Corn Flour Dependent on Postcooking Steeping Time. https://doi.org/10.1094/CCHEM.2002.79.1.162, Cereal Chem. 79(1), 162-166 (2002).

Fernández-Muñoz, J.L., Rojas-Molina, I., González-Dávalos, M. L., Leal, M., Valtierra, M.E., y San Martín-Martínez, E., Study of Calcium Ion Diffusion in Components of Maize Kernels During Traditional Nixtamalization Process. https://doi.org/10.1094/CCHEM.2004.81.1.65, Cereal Chem. 81(1), 65-69 (2004).

Fernández-Muñoz, J. L., San Martin-Martinez, E., Diaz-Gongora, J. A. I., Calderón, A., Alvarado-Escobar, A., OrtizCardenas, H., y Leal-Perez, M., Steeping time and cooking temperature dependence of calcium ion diffusion during microwave nixtamalization of corn. https://doi.org/10.1016/j.jfoodeng.2005.06.004, J. Food Eng. 76(4), 568-572 (2006)

Fernández-Muñoz, J. L., Gruntal-Santos, M. A., Hernández-Osorio, I., San Martin-Martínez, E., Calderón, A., DíazGóngora, J. A. I., y Aguilar-Santos, M. A., Estudio del proceso de secado en un secador de halógeno de los granos de maíz nixtamalizados. Superficies y Vacío, ISSN: 1665-3521 20 (4), 139-142 (2007).

Fernández-Muñoz, J.L., Zapata-Torrez, M., Márquez-Herrera, A., Sánchez-Sinencio, F., Mendoza-Álvarez, J. G., Meléndez-Lira, M., y Zelaya-Ángel O., "Properties of Particle Size Distribution from Milled White Nixtamalized Corn Kernels as a Function of Steeping Time. https://doi.org/10.1155/2016/6724047, Scientifica, vol. 2016, Article ID 6724047, 1-9, (2016)

Haladjian, N., Fayad, R., Toufeili, I., Shadarevian, S., Sidahmed, M., Baydoun, E., y Karwe, M., pH, temperature and hydration kinetics of faba beans. https://doi.org/10.1111/j.1745-4549.2003.tb00497.x, J. Food Processing and Preservation, 27, 9-20 (2003).

Giraldo-Zuniga, A. D., Arévalo-Pinedo, A., Silva, Ferreira-Silva, A., Valdes-Serra, J. C., y de Menezes Pavlak, M. C., Datos experimentales de la cinética del secado y del modelo matemático para pulpa de cupuaçu (Theobroma grandiflorum) en rodajas. https://doi.org/10.1590/S0101-20612010000100027, Food Science and Technology, 30(1), 179-182 (2010).

Gowen, A., Abu-Ghannam, N., Frias, J., y Oliveira, J., Influence of pre-blanching on the water absorption kinetics of soybeans. https://doi.org/10.1016/j.jfoodeng.2005.12.009, J. Food Eng. 78(3), 965-971 (2007).

Laria, J., Meza, E., Mondragon, M., Silva, R., y Pena, J.L., Comparison of overall water uptake by corn kernel with and without dissolved calcium hydroxide at room temperature. https://doi.org/10.1016/j.jfoodeng.2004.05.013, J. Food Eng. 67(4), 451-456 (2005)

Miano, C. A., Ibarz A., y Estevez-Duarte, A. P. E., Ultrasound technology enhances the hydration of corn kernels without affecting their starch properties. https://doi.org/10.1016/j.jfoodeng.2016.10.024, J. Food Eng. 197(1), 34-43 (2017).

Núñez-Santiago, M.C., Santoyo, E., Bello-Pérez, L.A., y Santoyo-Gutiérrez, S., Rheological evaluation of non-Newtonian Mexican nixtamalised maize and dry processed masa flours. https://doi.org/10.1016/S0260-8774(03)00018-9, J. Food Eng. 61(1), 55-66 (2003).

Peleg, M., An Empirical Model for the Description of Moisture Sorption Curves. https://doi.org/10.1111/j.13652621.1988.tb13565.x, J. Food Sci., 53(4), 1216-2017 (1988).

Ramírez-Araujo, H., Gaytán-Martínez, M., y Reyes-Vega, M. L., Alternative technologies to the traditional nixtamalization process: Review. https://doi.org/10.1016/j.tifs.2018.12.007, Trend Food Sci. Techn., 85(1), 34-43 (2019).

Ramírez-Jiménez, A. K., Rangel-Hernández, J., Morales-Sánchez, E., Loarca-Piña, G., y Gaytán-Martínez, M., Changes on the phytochemicals profile of instant corn flours obtained by traditional nixtamalization and ohmic heating process. https://doi.org/10.1016/j.foodchem.2018.09.166, Food Chem. 276, 57-62 (2019).

Rojas-Molina, I., Gutiérrez, E., Rojas, A., Cortés, M., Campos-Solís, L., Hernández-Urbiola, M., Arjona, J.L., Cornejo, A., y Rodríguez-García, M. E., Effect of Temperature and Steeping Time on Calcium and Phosphorus Content in Nixtamalized Corn Flours Obtained by Traditional Nixtamalization Process. https://doi.org/10.1094/CCHEM-86-5-0516, Cereal Chem. 86(5), 516-521 (2009).

SIAP/SAGARPA,2008,http://www.campomexicano.gob.mx/portalsiap/Integracion/EstadisticaDerivada/ComercioExterior/ Estudios/Perspectivas/maiz96-10.pdf.

Valderrama-Bravo, C., Rojas-Molina, A., Gutiérrez-Cortez, E., Rojas-Molina, I., Oaxaca-Luna, A., De la Rosa-Rincón, E., y Rodríguez-García, M.E., Mechanism of calcium uptake in corn kernels during the traditional nixtamalization process: Diffusion, accumulation and percolation. https://doi.org/10.1016/j.jfoodeng.2009.12.018, J. Food Eng. 98(1), 126-132 (2010).

VanCauwenberge, J.E, Bothast, R.J., y Kwolek, W.F., Thermal inactivation of eight Salmonella serotypes on dry corn flour, Appl. Environmental Microbiol, ISSN: 1098-5336, 42(4), 688-691 (1981). 
